\section{Going with the flow}

Michael A. Leschziner

International Journal of Computational Fluid Dynamics. Editor-in-chief W. G. Habashi. Gordon and Breach. 4/vol. ECU774, $\$ 930$ (institutional); ECU180, $\$ 196$ (personal). Two volumes in 1995.

COMPUTATIONAL fluid dynamics is one of several disciplines that have responded to developments in high-performance computers in much the same way as Californian and Texan boom towns reacted, around the turn of the century, to the discovery of gold and oil underfoot: a rapid, uncontrolled growth with very flaky edges, punctuated by tentacles sent out in all directions without a firm core infrastructure to support outlying areas. Matters have not been helped by the fact that the field, although a theoretically challenging branch of nonlinear mathematics and physics, is of outstanding practical importance in thermofluids engineering and has therefore been the victim of irresponsible exploitation. A further adverse consequence of the relevance of computational fluid dynamics to numerous industrial applications has been a proliferation of application-specific and often weakly founded variants of what are, essentially, sets of generic numerical tools that constitute the discipline. Recently, however, there has been an increasing realization that the key issues of computational fluid dynamics - among them numerical accuracy, resolution, computational economy and realism of the mathematical closures approximating physical processes - must be addressed or revisited in a systematic, focused and critical fashion.

This new journal wishes to be seen as distinct from others in being a specialized forum "serving the discipline as a unit and gathering under one title [its] main aspects". It declares its principal aims to include: "timely dissemination of innovative research ideas"; putting "emphasis on papers dealing with efficient methods to produce accurate predictive numerical tools for flow analysis"; being a "truly interdisciplinary forum" for computational fluid dynamics by publishing "latest advances in numerical methods in fluid dynamics for the aeronautics, astrophysics, environmental and process fields". These are all laudable objectives suggesting a firm intention to promote careful research on generic issues.

The first four issues do not suggest to the critical reader expecting a fresh approach that the journal is on its way to becoming a distinctive forum of the kind to which it aspires. The mix of articles does not distinguish itself dramatically from that in several other periodicals devoted to the field, and there does not seem to be a clear theme or a new philos- ophy behind the selection of contributions. Indeed, the reader is occasionally confronted by some startling subject sequences, moving, for example, from supersonic flow by way of the analysis of data on surface-water quality to turbulence modelling. Although several articles are strong and present impressive achievements, especially in finite element methods, some are mediocre, and a few are rather weak. In at least two instances, complex flows are analysed with firstorder convection schemes, which gives no credit to the papers or the journal. Few of the papers include statements on numerical accuracy, grid-independence and error bounds - the inclusion of these is a must for any journal laying claim to rigour. On a more mundane level, the linguistic quality of some papers is not high, and the quality of graphical material is variable. Also, there appears to be no uniformity in the nomenclature, the inclusion or exclusion of keywords or even in the use of the heading "Abstract" or "Summary" at the beginning of articles. A pleasing feature of the journal, however, is that it permits "Short Communications", thus promoting rapid dissemination of new developments.

In summary, unless, it gains a more distinct profile, the journal will establish itself as only one of several alternative outlets for research on computational fluid dynamics. At present, there continues to be a gap in the 'CFD market' for a specialist journal with a fresh approach that places emphasis on rigour and on carefully verified improvements to the generic constituents of the discipline.

Michael A. Leschziner is in the Department of Mechanical Engineering, University of Manchester Institute of Technology, PO Box 88, Manchester M60 1QD, UK.

\title{
Listening while learning
}

\section{Sanjiva K. Lele}

Journal of Computational Acoustics. EdiWorld Scientific. 4/yr. $\$ 190$ (institutions); $\$ 95$ (personal).

ACCORDING to the editors of Journal of Computational Acoustics, the traditional compartmentalization of acoustics and related branches of science and engineering has not been conducive to the exchange of ideas and information. This new journal intends to provide scientists and engineers - whether their main interest is in oceanography, aerodynamics, applied mathematics, noise control, meteorology, geophysics, bioengineering, nondestructive testing, mechanical systems or computer science - with a forum for sharing their achievements in, and insights into, the broad field of computational acoustics. The authorship of early issues reflects these diverse communities.

But why limit the potential of this cross-fertilization of ideas? Why restrict coverage to computational acoustics? tors-in-chief Ding Lee and Allen D. Pierce.

There is perhaps a feeling that recent developments in computer hardware and numerical algorithms will allow a new computational assault on a wide range of problems in acoustics. Computational tools for acoustical analysis and design with respect to structural engineering are also on the horizon. And there is increasing concern about noise pollution, leading to a push for new technologies that are quieter or capable of controlling noise.

Computational methods may well help to develop and perfect these technologies, but their precise role is difficult to predict. Some areas may require an engineer's blend of theory, experiment and computation. The journal does have a niche, but its effectiveness will depend on whether it can encourage a proper dialogue among the applied acoustics communities it aims to embrace.

Sanjiva $K$. Lele is in the Department of Aeronautics and Astronautics and Mechanical Engineering, Stanford University, Stanford, California 94305-4035, USA

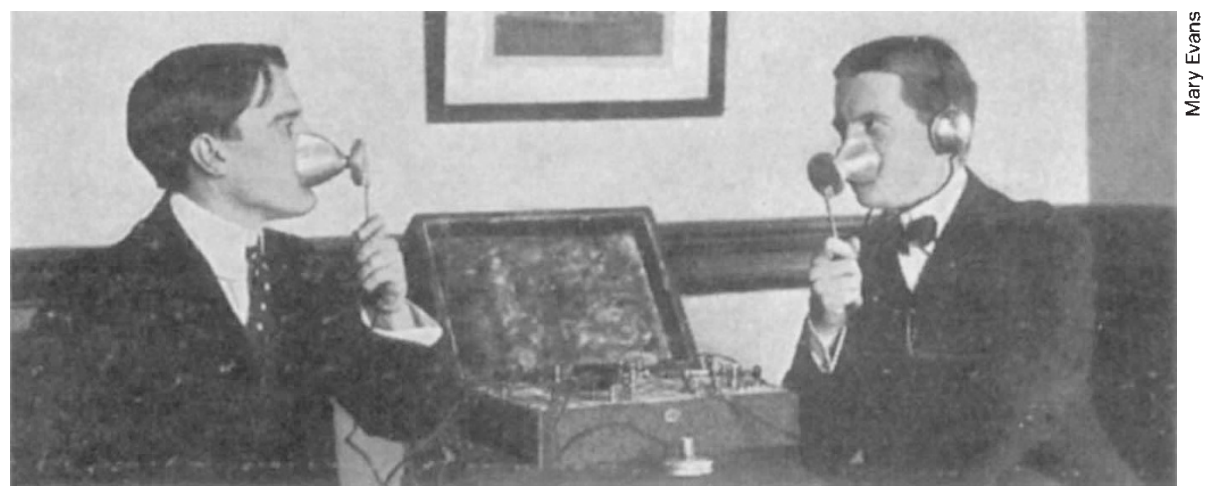

The "Acousticon", an instrument enabling the deaf to hear, was invented by M. R. Hutchinson in 1903. He used it successfully on thousands of deaf mutes in New York. 